\title{
Raman Spectroscopic Evidence of a Coherent Room Temperature Hybrid Structure of $\mathrm{BaTiO}_{3}$ Single Crystal
}

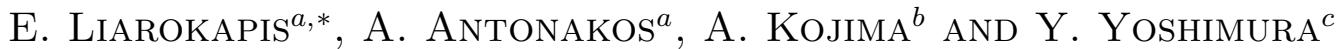 \\ ${ }^{a}$ Department of Physics, National Technical University, GR-15780 Athens, Greece \\ ${ }^{b}$ Department of Material Science, The University of Shiga Prefecture, Hikone, Shiga 522-8533, Japan \\ ${ }^{c}$ Faculty of Science and Engineering, Ritsumeikan University, Kyoto, Shiga 522-8533, Japan
}

Recently, a precession X-ray study has discovered that ferroelectric $\mathrm{BaTiO}_{3}$ single crystals have a coherent hybrid structure that consists of tetragonal and monoclinic lattices that share the (101) face of the tetragonal $[11,12]$. Microscopic observations attest that the single crystal has two kinds of stripes (dark and white regions). Raman spectra of all sides of the cubic crystal have been collected at several scattering configurations. Two kinds of domains have been observed by the Raman measurements within the stripes, which are identified as light and dark spots under the optical microscope. The spectra structures and line widths are strongly affected when the laser direction is parallel to the $b$-axes and the polarization parallel to the $a$ - or $c$-axes of the $90^{\circ}$ domain ( $a c$-side). It appears that there is a symmetry reduction and disorder, which affects the Raman active phonons. The results are in agreement with the coexistence of the two (tetragonal and monoclinic) lattices.

PACS numbers: 78.30.-j, 77.80.Dj, 63.50.- -

\section{Introduction}

Barium titanate $\left(\mathrm{BaTiO}_{3}\right)$ has dominated the ferroelectric field for more than 60 years as the most suitable object for many researchers to make both fundamental and application studies. Accordingly, huge amount of experimental and theoretical studies relevant to $\mathrm{BaTiO}_{3}$ have been made up to now for the purpose of clarifying the origin of ferroelectricity. As for the past studies of the ferroelectric transition, however, there exists the well-known controversy between two opposite interpretations of the phase transition mechanism depending on their experimental methods, the displacement and the order-disorder type, but it has been left until today without any conclusive result. Light scattering has been used to investigate the zone-centered phonons [1] and check the soft mode [2] across the phase transitions. The phase transition between the ferroelectric and the paraelectric phase has been studied as a function of temperature, using both Raman and infrared spectroscopic measurements [3]. In addition, there are some Raman measurements as a function of pressure [4, 5].

Recently, a "mK-stabilized cell" has been developed [6-9] capable of observing the phase transition itself by enlarged temperature scale. The phase transition of the $\mathrm{BaTiO}_{3}$ single crystal observed at $403 \mathrm{~K}$ by the "mK-stabilized cell" has brought very significant results; the process on heating is quite simple having a single thermal anomaly but the process on cooling has two ther-

\footnotetext{
* corresponding author
}

mal anomalies. In other word, the ferroelectric phase transition is not reversible but irreversible [10]. Motivated by the stimulating results of the phase transition on cooling, X-ray diffraction studies have been performed in the ferroelectric room temperature phase [11], using $\mathrm{BaTiO}_{3}$ single crystals. The result is notable: the ferroelectric phase has the coherent hybrid structure (CHS) composed of tetragonal and monoclinic forms [11, 12], opposite to the common today knowledge. However there comes about a question concerning its materialization in the bulk single crystal.

In the present study we report micro-Raman measurements on the $\mathrm{BaTiO}_{3}$ single crystals, in order to examine the hybrid structure that has been discovered by the systematic X-ray diffraction (XRD) studies.

\section{Experimental details}

$\mathrm{BaTiO}_{3}$ single crystals made by the top-seeded solution growth method were used for the present studies. They are very fine single crystals served by MTI Co. in USA and might be called "photoreflective grade" if it were poled. Figure 1 shows a photograph of a cubed sample with the size of $4 \times 4 \times 4 \mathrm{~mm}^{3}$, which is cut along the three crystallographic directions and etched by phosphoric acid to remove some strain caused by cutting from an as-grown boule.

Micro-Raman spectra were acquired at room temperature with a Jobin-Yvon T64000 Raman system, using a $514.5 \mathrm{~nm} \mathrm{Ar}{ }^{+}$laser, in a backscattering geometry. The scattering configuration for each spectrum is indicated, where the first (second) symbol defines the polarization 


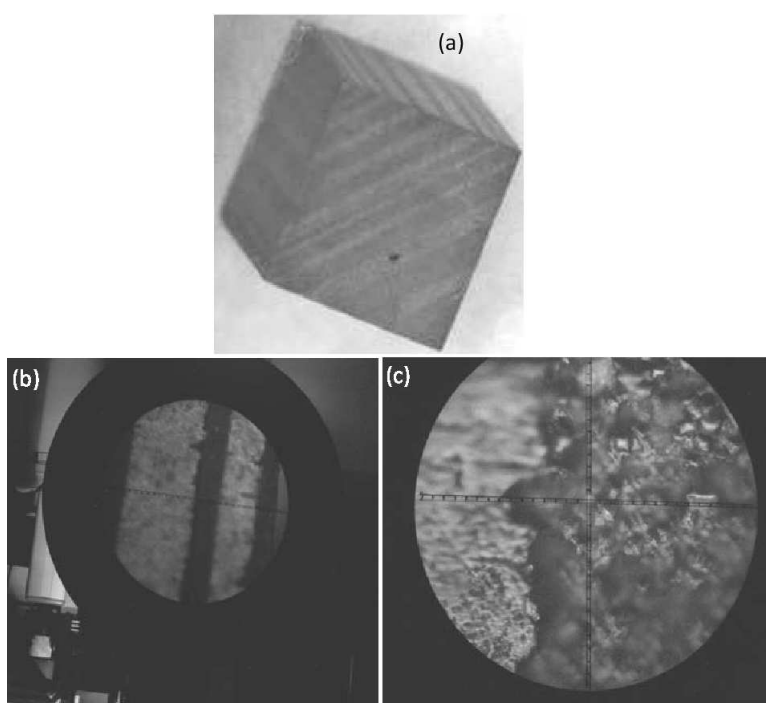

Fig. 1. (a) Photograph of the cubed $\mathrm{BaTiO}_{3}$ single crystal. The so-called $90^{\circ}$ domain pattern having oblique stripes with interstitial dark and bright colors is clearly observed on the top surface of the sample even by the naked eyes. The $90^{\circ}$ domain pattern under the white light of the metallurgical microscope and (b) the $40 \times$ magnification and (c) the $100 \times$ magnification.

of the incident (scattered) light. A $40 \times$ lens, giving an approximate laser spot diameter of $4 \mu \mathrm{m}$ was mainly used for data accumulation at a power of $0.10-0.15 \mathrm{~mW}$ to ensure minimum disturbance for the $4 \times 4 \times 4 \mathrm{~mm}^{3}$ crystal. Under the white light of the metallurgical microscope (also kept at low intensity) the $90^{\circ}$ domain pattern appears as luminous (white) and dark stripes of varying width 100-150 $\mu \mathrm{m}$ (Fig. 1b). By increasing the magnification to $100 \times$ (Fig. 1c), one observes that the stripes consist of needle-like white and dark islands (domains) oriented along the axes. Thus, the only difference between the two kind of stripes is the relative density of the dark and white islands. The dark and white islands do not change or exchange colors upon rotation of their orientation under the microscope white light.

Raman spectra were obtained from various scattering polarizations and from several islands in each stripe. In order to investigate the depth of those islands we have acquired spectra at various magnification levels and by using a pinhole located close to the entrance to the spectrometer.

\section{Results and discussion}

Typical Raman spectra from the oblique side of the crystal are seen in Figs. 2a, b. Figure 2a shows the spectra of the white and dark islands. The stronger differences are observed in the $b(c c) b$ scattering geometry and are (a) the appearance of a broad continuum in the region $190-290 \mathrm{~cm}^{-1}$ for the dark islands and (b) a relative intensity redistribution of the multicomponent $\approx 500 \mathrm{~cm}^{-1}$ feature. Figure $2 \mathrm{~b}$ compares spectra from dark islands in
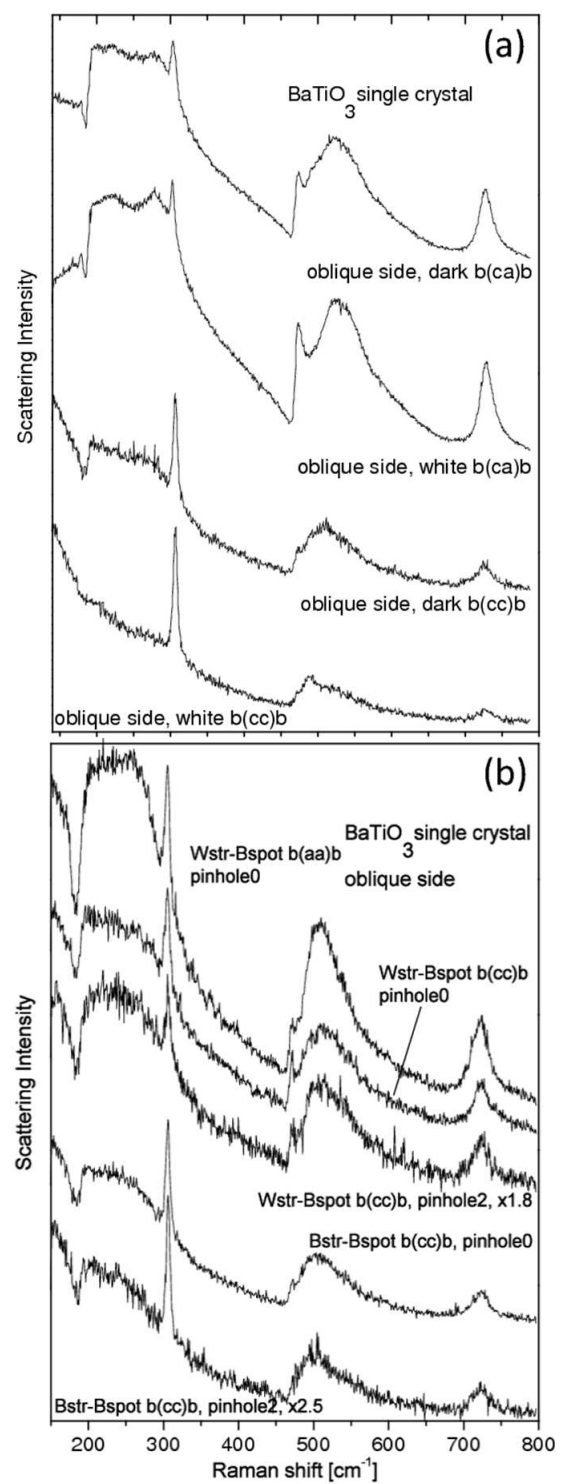

Fig. 2. (a) Typical Raman spectra from the white (luminous) and dark islands on the oblique side of the crystal, acquired in the $\bar{b}(c c) b$ and $\bar{b}(c a) b$ scattering geometry. (b) Comparison of spectra from dark islands in the white and dark stripes and their dependence on the spectrometer pinhole diameter (pinhole $0=$ fully open $>$ pinhole 2).

the white and dark stripes and their dependence on the spectrometer pinhole: the orientation of the dark "phase" does not seem to change between the two types of stripes (although the 190-290 $\mathrm{cm}^{-1}$ continuum is stronger in the geometry) and the pinhole diameter (pinhole $0=$ fully open $>$ pinhole 2) does not induce significant differences. As for the $90^{\circ}$ domains, however, it has been assumed until today that they are interstitially composed of the same kind of a tetragonal domain structure having perpendicularly polarized directions so that the color difference is due to the direction of the polarization. This 
may not be correct from the fact that the domain colors do not always change mutually in case of a $90^{\circ}$ rotation, since we have not observed any polarization difference between the two kinds of stripes or between the luminous (or dark) islands. By the present micro-Raman data in Fig. 2, it has been clarified that the two kinds of domains of the $90^{\circ}$ domain are not $100 \%$ pure domains but have some admixture of the other domain as well. The Raman data prove that the luminous islands are more crystalline than the dark ones, which appear more disordered. The preferential creation of such islands and their orientation with respect to the axes must be related with the formation of the domain boundaries.
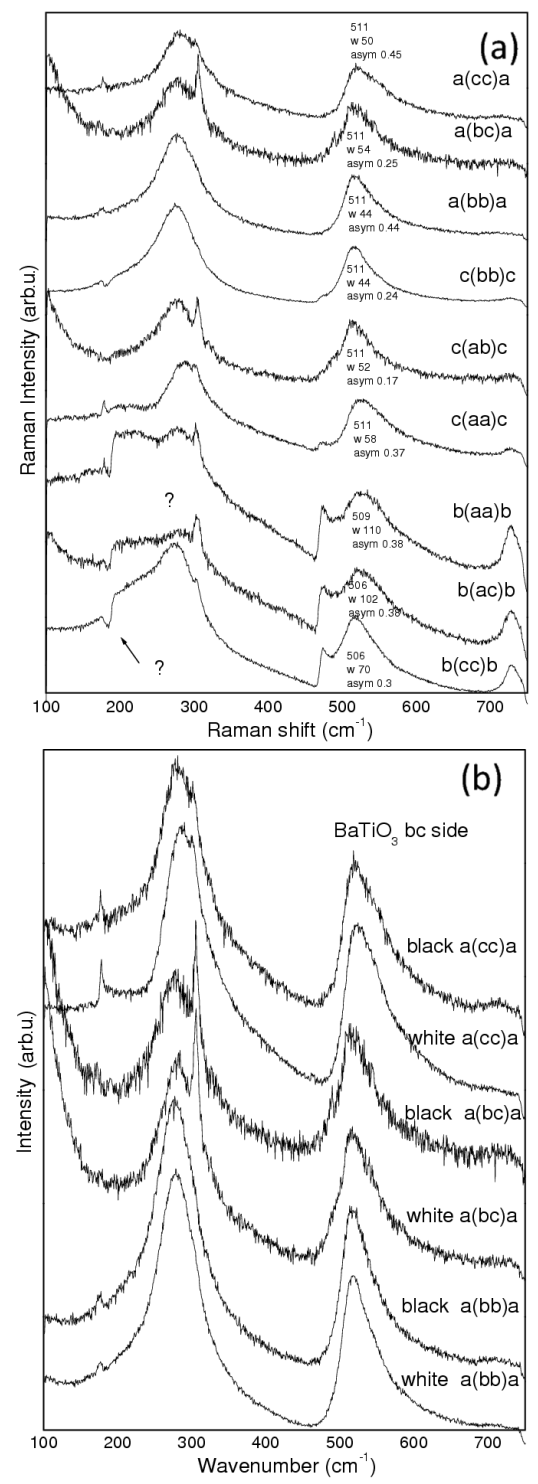

Fig. 3. (a) Polarized Raman spectra measured on the $\mathrm{BaTiO}_{3}$ single crystal. The energy, the width (w), and the Fano asymmetry (asym) of the $\approx 500 \mathrm{~cm}^{-1}$ band are indicated in $\mathrm{cm}^{-1}$. (b) Polarized Raman spectra of the $b c$ side of the $\mathrm{BaTiO}_{3}$ single crystal. The most noticeable difference between the dark and the white domains is the signal to noise $(\mathrm{S} / \mathrm{N})$ ratio.
In Fig. 3a the spectra have been collected on different scattering geometries in order to check the orientation of the crystal. The bands at $180 \mathrm{~cm}^{-1}, 302 \mathrm{~cm}^{-1}$, $470 \mathrm{~cm}^{-1}$ and $715 \mathrm{~cm}^{-1}$ have been observed before $[1,5]$ and have the $A_{1}(\mathrm{TO}), B_{1}, A_{1}(\mathrm{TO}), E$-symmetry, respectively. The broad bands at $\approx 270 \mathrm{~cm}^{-1}$ and $\approx 520 \mathrm{~cm}^{-1}$ have been associated with 2 nd order scattering $[1,5]$. The strong enhancement of those bands on certain scattering configurations from the dark islands indicate strong lattice distortions. Since all modes are related with the oxygen vibrations, it is reasonable to assume a stronger oxygen disorder in the dark islands. The XRD results indicate that the oxygen disorder is more pronounced in the tetragonal phase [13]. Therefore, we conclude that the dark islands and stripes should mostly consist of the tetragonal structure and the white ones from the monoclinic.

As Fig. 3b presents, the white stripes and the black stripes spectra with the $10 \times$ magnification are more or less the same. There is only a difference in the intensity and the $\mathrm{S} / \mathrm{N}$ ratio between the white and black stripes spectra. Bright domains have better $\mathrm{S} / \mathrm{N}$ than their adjacent dark domains, which could be also attributed to a structural disorder. So, it is guessed that the bright domains are mainly composed of the monoclinic structure while the dark domains of the tetragonal because the monoclinic structure has less oxygen vacancies than the tetragonal structure. In addition, according to [5], in the Raman spectra of the rhombohedral phase that appears at low temperatures, the intensity of the same bands is increased and their shapes are modified. Therefore, the oxygen disorder model is supported by the Raman study by the difference of $\mathrm{S} / \mathrm{N}$ of the peaks.

In conclusion, the above results agree with the hybrid structural model as discovered by the XRD measurements. The Raman data indicate an increased disorder in the black islands as compared with the white ones. The amount of the black islands in the two kinds of stripes is different, which creates the different brightness of the stripes.

\section{Acknowledgments}

Work partially supported by the E.C. STREP project COMEPHS.

\section{References}

[1] D.A. Tenne, A. Soukiassian, X.X. Xi, H. Choosuwan, R. Guo, A.S. Bhalla, Phys. Rev. B 70, 174302 (2004) and references therein.

[2] P. Anderson, in: Proc. All-Union Conf. on the Physics of Dielectrics, Acad. Sci. USSR, Moscow 1958, p. 209; W. Cochran, Adv. Phys. 9, 387 (1960) and 10, 401 (1961); H. Vogt, J.A. Sanjurio, G. Rossbroich, Phys. Rev. B 26, 5904 (1982).

[3] A. Chaves, R.S. Katiyar, S.P.S. Porto, Phys. Rev. B 10, 3522 (1974); A. Scalabrin, A.S. Chaves, D.S. Shim, S.P.S. Porto, Phys. Status Solidi 79, 731 (1977); J.A. Sanjurjo, R.S. Katiyar, S.P.S. Porto, Phys. Rev. B 22, 2396 (1980). 
[4] L.-G. Liu, W.A. Bassett, Elements, Oxides, and Silicates, Oxford Univesity Press, New York 1986.

[5] U.D. Venkateswaran, V.M. Naik, R. Naik, Phys. Rev. B 58, 14256 (1998).

[6] A. Kojima, C. Ishii, K. Tozaki, S. Matsuda, T. Nakayama, N. Tsuda, Y. Yoshimura, H. Iwasaki, Rev. Sci. Instrum. 68, 2301 (1997).

[7] K. Tozaki, C. Ishii, O. Izuhara, N. Tsuda, Y. Yoshimura, H. Iwasaki, Y. Noda, A. Kojima, Rev. Sci. Instrum. 69, 3298 (1998).

[8] Y. Yoshimura, A. Kojima, T. Yoshioka, Y. Kawakatsu, K. Tozaki, H. Iwasaki, Meas. Sci. Technol. 12, L5 (2001).

[9] K. Tozaki, T. Okazaki, A. Kojima, Y. Yoshimura, Rev. Sci. Instrum. 76, 066104 (2005).
[10] A. Kojima, H. Sasou, K. Tozaki, T. Okazaki, Y. Yoshimura, N. Tokunaga, H. Iwasaki, Int. J. Thermophys. 26, 1963 (2005).

[11] Y. Yoshimura, A. Kojima, N. Tokunaga, K. Tozaki, T. Koganezawa, Phys. Lett. A 353, 250 (2006).

[12] Y. Yoshimura, M. Morioka, A. Kojima, N. Tokunaga, T. Koganezawa, K. Tozaki, Phys. Lett. A 367, 394 (2007).

[13] Y. Yoshimura, M. Morioka, A. Kojima, to be submitted. 